\title{
ATLAS: B Physics Reach
}

EP Jdirect
electronic only

(C) Springer-Verlag 2003

\author{
M. Smizanska, Lancaster University, UK \\ On behalf of the ATLAS collaboration \\ Received:
}

\begin{abstract}
The current scope and status of the ATLAS B-physics trigger and of offline performance studies are presented. From the initial running at low-luminosity at LHC, high-statistics B analyses will allow sensitive tests of possible new physics contributions by searching for additional $\mathrm{CP}$ violating effects and for anomalous rates of rare B-decay channels. In the physics of the $\mathrm{B}_{\mathrm{s}}^{0}$ meson system there is sensitivity to mass and width differences and to a weak mixing phase beyond the Standard Model expectation. ATLAS will also be able to access rare B decays using the high-luminosity running. Measurements of beauty production sensitive to higher order QCD terms will also be made, providing new data to investigate present inconsistencies between theory and experiment.
\end{abstract}

PACS: 25.70.Ef; 21.60.Gx; 27.30.+t

\section{Introduction}

In recent years B-physics received a lot of attention and presently the measurements of B-factories are being published, see for instance [1, 2]. CDF and D0 have already started very promising measurements in RUN-II [3, 4]. Although the physics potential of these experiments is high, it may well be that the very precise B-decay measurements needed for finding evidence of new physics will be left for the LHC experiments. The LHC should also make possible QCD tests in beauty production in proton-proton collisions at the highest energies.

ATLAS has recently addressed the issue of maximizing B-physics capabilities in the context of a possibly reduced initial Trigger and Data Acquisition (DAQ) system. While these studies are still ongoing, the present paper separates the off-line performance results into two categories; the first one uses the full trigger potential while the other group of results is given for a minimal-trigger scenario with only a di-muon trigger. Although it is clear that the luminosity will vary during the initial LHC period and during fills, all the results are given for a common integrated luminosity $30 \mathrm{fb}^{-1}$ corresponding to 3 years running of a luminosity $10^{33} \mathrm{~cm}^{-2} \mathrm{~s}^{-1}$. In the case of rare decays, $\mathrm{B}_{\mathrm{d}} \rightarrow \mu^{+} \mu^{-}(\mathrm{X})$, results are also given for one year at $10^{34} \mathrm{~cm}^{-2} \mathrm{~s}^{-1}$. The ATLAS program both for B-decays and for beauty production is covered.

\section{B-physics triggers}

In ATLAS about one collision in every hundred will produce a b $\bar{b}$ quark pair. Therefore, in addition to rejecting non-b $\bar{b}$ events, the B-trigger must have the 
(a)
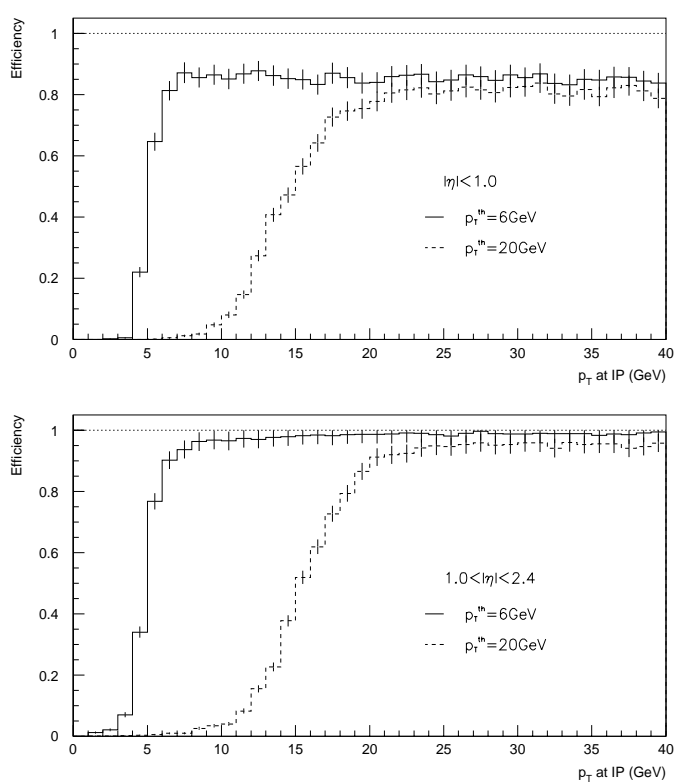

(b)

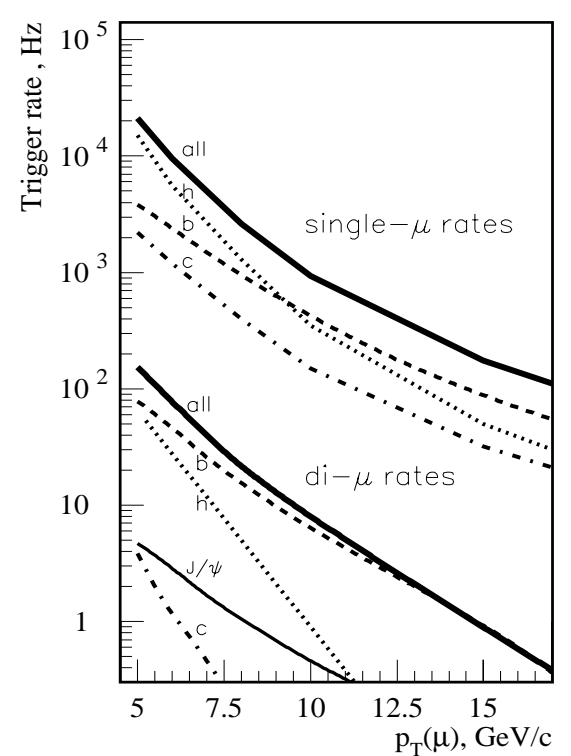

Fig. 1. (a) First level muon trigger efficiency as a function of muon $p_{\mathrm{T}}$ in the barrel and endcap parts of the trigger chambers. The lower threshold of $6 \mathrm{GeV}$ is a minimal $p_{\mathrm{T}}$ option for B-physics. (b) Single and di-muon trigger rates for luminosity $10^{33} \mathrm{~cm}^{-2} \mathrm{~s}^{-1}$. Muons coming from decays of $\mathrm{K}^{ \pm}$or $\pi \pm$ are denoted by a letter ' $h$ ', those from beauty decays by a letter ' $b$ ', from charm by a letter 'c'.

ability to identify and select those events containing B-decays channels of specific interest. Recent work [5] has addressed the issue of maximizing B-physics capabilities in the context of developments, such as the likelihood of a reduced inner detector layout at the start of running, various start-up luminosity scenarios and a possibly reduced initial Trigger/DAQ system.

A flexible trigger strategy has been developed based on a di-muon trigger at the start of higher luminosity $\left(2 \cdot 10^{33} \mathrm{~cm}^{-2} \mathrm{~s}^{-1}\right)$ LHC fills and introducing further triggers later in the beam coast and for low luminosity fills. The additional triggers will require a jet or an electromagnetic cluster to be identified in addition to a single muon by the first level-1 trigger. All level-1 objects will guide reconstruction at level-2 and the results of the level-2 trigger will seed reconstruction in the Event Filter. Performing the reconstruction in only a limited region of the inner detector containing a $\mathrm{B}$ candidate strongly reduces the processing power requirements compared to the previous strategy that involved a full scan of the inner detector at level-2 [6].

The di-muon trigger provides a very effective selection of several important 
channels, e.g. $\mathrm{B}_{\mathrm{d}} \rightarrow \mathrm{J} / \psi\left(\mu^{+} \mu^{-}\right) \mathrm{K}_{\mathrm{s}}^{0}, \mathrm{~B}_{\mathrm{s}}^{0} \rightarrow \mathrm{J} / \psi\left(\mu^{+} \mu^{-}\right) \phi$ and $\mathrm{B}_{\mathrm{d}} \rightarrow \mu^{+} \mu^{-}(\mathrm{X})$. The level-1 muon trigger is efficient down to a transverse momentum $p_{\mathrm{T}}$ of about $5 \mathrm{GeV}$ in the barrel and about $3 \mathrm{GeV}$ in the endcaps, see Fig.1a. However the actual thresholds will be determined by rate limitations. For example at a luminosity of $10^{33} \mathrm{~cm}^{-2} \mathrm{~s}^{-1}, p_{\mathrm{T}}$ thresholds of $6 \mathrm{GeV}$ and $5 \mathrm{GeV}$ for the first and second muons in the event respectively would give a trigger rate of $180 \mathrm{~Hz}$ after sharp muon $p_{\mathrm{T}}$ cuts applied at second level trigger, see Fig.1b. These triggers are mostly due to muons from beauty decays with a small contribution from events with one muon from a $\mathrm{K}^{ \pm}$or $\pi^{ \pm}$decay in flight.

The jet-muon trigger will cover hadronic final states. They arise from processes such as $\mathrm{B}_{\mathrm{s}}^{0} \rightarrow \mathrm{D}_{\mathrm{s}} \pi, \mathrm{B}_{\mathrm{s}}^{0} \rightarrow \mathrm{D}_{\mathrm{s}} \mathrm{a}_{1}$ and $\mathrm{B}_{\mathrm{d}} \rightarrow \pi^{+} \pi^{-}$. All such processes will contain a muon identified by first level trigger and confirmed at level two, which will be used to tag the B-hadron flavour. The electron-muon trigger is used to select decay channels such as $\mathrm{B}_{\mathrm{d}} \rightarrow \mathrm{J} / \psi\left(\mathrm{e}^{+} \mathrm{e}^{-}\right) \mathrm{K}_{\mathrm{s}}^{0}$ or $\mathrm{B}_{\mathrm{d}} \rightarrow \mathrm{J} / \psi\left(\mu^{+} \mu^{-}\right) \mathrm{K}_{\mathrm{s}}^{0}$ with the tags from the semileptonic decays of associated B-hadrons to a muon or an electron respectively.

The ATLAS trigger system output rate for beauty physics is expected to be $\sim 10 \mathrm{~Hz}$ which corresponds to about $10^{8}$ events with beauty hadron decays delivered to permanent storage.

\section{$3 \quad$ Selected detector performance characteristics}

The ATLAS detector has been primarily designed to search for new particles at the highest mass scales; however B-physics requirements have been accommodated in the design. ATLAS allows clean lepton identification over a wide range of transverse momenta. Electrons can be identified from $p_{\mathrm{T}}$ values as low as $\sim 0.5 \mathrm{GeV}$ in the transition radiation tracker, the outer part of the ATLAS inner detector tracking system. An example in Fig.2a, taken from [7], shows $\mathrm{J} / \psi \rightarrow \mathrm{e}^{+} \mathrm{e}^{-}$background rejection for $\mathrm{B}_{\mathrm{d}} \rightarrow \mathrm{J} / \psi\left(\mathrm{e}^{+} \mathrm{e}^{-}\right) \mathrm{K}_{\mathrm{s}}^{0}$ events. For electrons with $p_{\mathrm{T}}$ higher than $\sim 4 \mathrm{GeV}$, an additional identification comes from the electromagnetic calorimeter, placed beyond the solenoid coil surrounding the inner detector. Electrons with $p_{\mathrm{T}}>5 \mathrm{GeV}$ can be used to tag the B-hadron flavour. Using a combined identification of the inner detector and the calorimeter, $70 \%$ of inclusive $\mathrm{b} \overline{\mathrm{b}} \rightarrow \mu \mathrm{eX}$ events can be reconstructed with an efficiency of $1 / 570$ for background events, $\mathrm{b} \overline{\mathrm{b}} \rightarrow \mu \mathrm{X}$, not containing an electron. At the level-2 trigger with a luminosity of $10^{33} \mathrm{~cm}^{-2} \mathrm{~s}^{-1}$ this corresponds to a muon-electron trigger rate of $50 \mathrm{~Hz}$ containing only $10 \mathrm{~Hz}$ of b $\overline{\mathrm{b}} \rightarrow \mu \mathrm{X}$ background.

Good muon performance in ATLAS is achieved by combining information from the precision muon chambers with that of the inner detector. Muon reconstruction is efficient down to a $p_{\mathrm{T}}$ of about $6 \mathrm{GeV}$ in the barrel and about $3 \mathrm{GeV}$ in the endcaps (Fig.3a). Low $p_{\mathrm{T}}$ muon reconstruction can be increased by including barrel parts of hadron calorimeter [8]. A study made for $\mathrm{B}_{\mathrm{d}} \rightarrow \mathrm{J} / \psi\left(\mu^{+} \mu^{-}\right) \mathrm{K}^{0}$ events showed, Fig. 3b, that the mass reconstruction is not degraded even in cases where the $p_{\mathrm{T}}$ of $\mathrm{b}$ quark is larger than $50 \mathrm{GeV}$ [10], where the matching of tracks between the muon chambers and the inner detector is more difficult. 
(a)

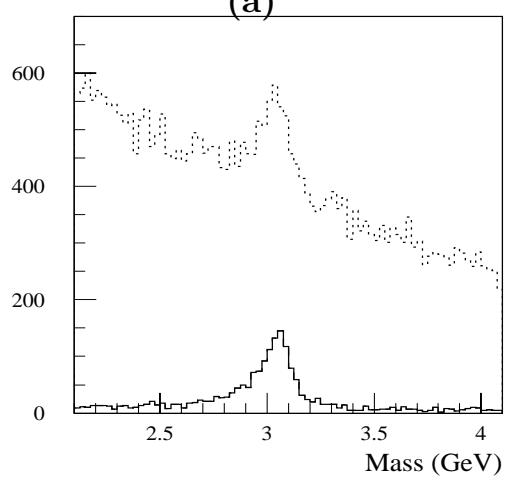

(b)

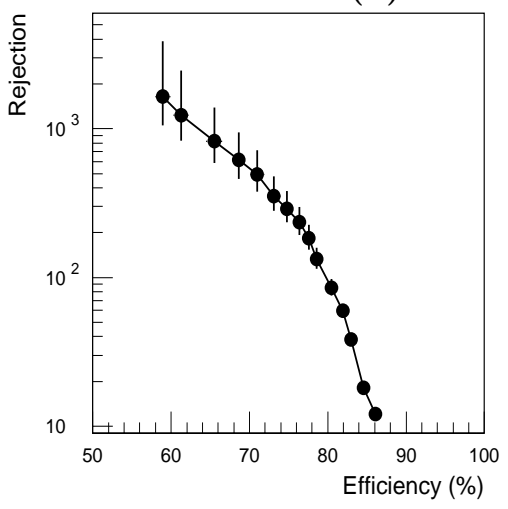

Fig. 2. (a) Rejection of combinatorial background using electron identification in Transition Radiation Tracker. Invariant mass of all track combinations in events containing $\mathrm{J} / \psi \rightarrow \mathrm{e}^{+} \mathrm{e}^{-}$before (dashed) and after (solid) identification cuts. (b) Rejection of $\mathrm{b} \overline{\mathrm{b}} \rightarrow \mu \mathrm{X}$ events without electron vs efficiency of selection of semiinclusive events $\mathrm{b} \overline{\mathrm{b}} \rightarrow \mu \mathrm{eX}$.

(a)

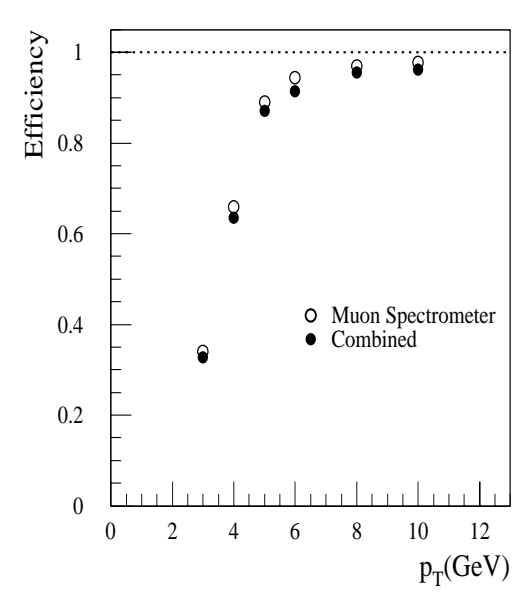

(b)

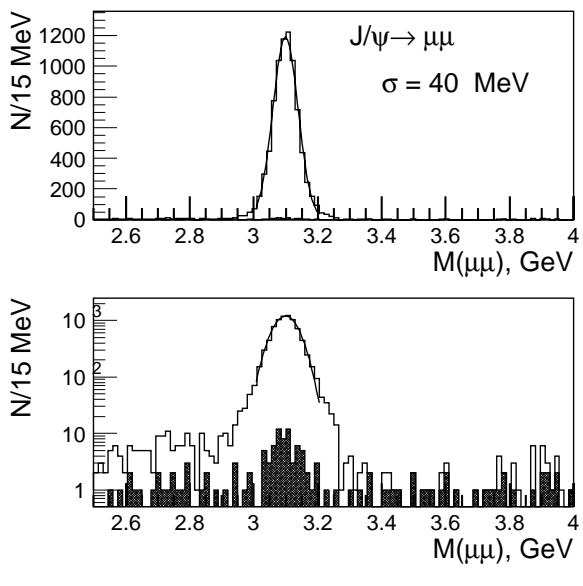

Fig. 3. (a) Efficiency of muon off-line reconstruction using the muon precise chambers and the inner detector as a function of muon $p_{\mathrm{T}}$. (b) The $\mathrm{J} / \psi$ mass reconstruction in $\mathrm{B}_{\mathrm{d}} \rightarrow \mathrm{J} / \psi\left(\mu^{+} \mu^{-}\right) \mathrm{K}^{0}$ events where the $p_{\mathrm{T}}$ of $\mathrm{b}$ quark is larger than $50 \mathrm{GeV}$ (linear and logarithmic scales). In part of events a muon detected in the muon system was associated with the wrong track in the inner detector (black area). 


\begin{tabular}{|c|c|c|c|}
\hline & $\mathrm{J} / \psi(\mu 6 \mu 3)$ & $\mathrm{J} / \psi(\mu 6 \mu 5)$ & $\mathrm{J} / \psi(\mathrm{e} 1 \mathrm{e} 1)$ \\
\hline Number of reconstructed events & $\overline{490 \mathrm{k}}$ & $250 \mathrm{k}$ & $15 \mathrm{k}$ \\
\hline Signal/background & 28 & 32 & 16 \\
\hline$\delta \sin (2 \beta)_{\text {stat }}$ lepton tag & 0.023 & 0.030 & 0.018 \\
\hline$\delta \sin (2 \beta)_{\text {stat }}$ jet-charge tag & 0.015 & 0.019 & - \\
\hline$\delta \sin (2 \beta)_{\text {stat }}$ combined tag & 0.0126 & 0.016 & 0.018 \\
\hline \multirow{2}{*}{\multicolumn{3}{|c|}{$\delta \sin (2 \beta)_{\text {stat }} \mathrm{J} / \psi(\mu 6 \mu 3)+\mathrm{J} / \psi(\mathrm{e} 1 \mathrm{e} 1)$}} & 0.010 \\
\hline & & & 0.016 \\
\hline
\end{tabular}

Table $1 . \mathrm{B}_{\mathrm{d}} \rightarrow \mathrm{J} / \psi \mathrm{K}_{\mathrm{s}}^{0}$ - summary of the expected event yields after 3 years of a luminosity $10^{33} \mathrm{~cm}^{-2} \mathrm{~s}^{-1}$ and the results obtained with different tagging methods and trigger selections. The trigger $p_{\mathrm{T}}$ thresholds in $\mathrm{GeV}$ are indicated in brackets.

\section{Precise $\sin (2 \beta)$ measurement from $\mathrm{B}_{\mathrm{d}} \rightarrow \mathrm{J} / \psi \mathrm{K}_{\mathrm{s}}^{0}$}

ATLAS sensitivity to the $\sin (2 \beta)$ measurement with the $\mathrm{B}_{\mathrm{d}} \rightarrow \mathrm{J} / \psi \mathrm{K}_{\mathrm{s}}^{0}$ decay was estimated with the maximum likelihood method. The detector performance parameters used as an input to the maximum likelihood analyses were first determined from the detector simulation and reconstruction of the $\mathrm{B}_{\mathrm{d}} \rightarrow \mathrm{J} / \psi \mathrm{K}_{\mathrm{s}}^{0}$ signal and of corresponding backgrounds. The likelihood function has the form:

$$
L=\prod_{i=1}^{N} \int_{0}^{\infty} \frac{\left(\epsilon_{1} W^{+}\left(t_{i}\right)+\epsilon_{2} W^{-}\left(t_{i}\right)+b \mathrm{e}^{-\Gamma_{0} t_{i}}\right) \rho\left(t-t_{i}\right) \mathrm{d} t}{\int_{t_{\min }}^{\infty}\left(\int_{0}^{\infty}\left(\epsilon_{1} W^{+}(t)+\epsilon_{2} W^{-}(t)+b \mathrm{e}^{-\Gamma_{0} t}\right) \rho\left(t^{\prime}-t\right) \mathrm{d} t^{\prime}\right) \mathrm{d} t}
$$

with

$$
W_{ \pm}(t)=e^{-\Gamma t} \Gamma\left(1+|\mathcal{A} / \overline{\mathcal{A}}|^{2} \mp\left(1-|\mathcal{A} / \overline{\mathcal{A}}|^{2}\right) \cos (\Delta \mathrm{mt}) \mp \sin (2 \beta) \sin (\Delta \mathrm{mt})\right)
$$

where $\mathrm{W}_{+}(\mathrm{t})$ and $\mathrm{W}_{-}(\mathrm{t})$ are time evolving probability functions describing the decays $\mathrm{B}_{\mathrm{d}} \rightarrow \mathrm{J} / \psi \mathrm{K}_{\mathrm{s}}^{0}$ and $\overline{\mathrm{B}_{\mathrm{d}}} \rightarrow \mathrm{J} / \psi \mathrm{K}_{\mathrm{s}}^{0}$ respectively, $\mathcal{A}$ and $\overline{\mathcal{A}}$ are complex amplitudes of the $\mathrm{B}_{\mathrm{d}} \rightarrow \mathrm{J} / \psi \mathrm{K}_{\mathrm{s}}^{0}$ and $\overline{\mathrm{B}_{\mathrm{d}}} \rightarrow \mathrm{J} / \psi \mathrm{K}_{\mathrm{s}}^{0}$ decays at time $t=0$. The meaning of other symbols in equations (1) and (2) is as follows: w is the wrong tag fraction, $\epsilon_{1}=1-w, \epsilon_{2}=w$ for the case in which the $\mathrm{B}$ is tagged as a particle, and $\epsilon_{1}=w, \epsilon_{2}=1-w$ for the case in which the B is tagged as an antiparticle, $b$ is the level of the background and $\Gamma_{0}$ is the average decay rate of background as determined from simulation. The time resolution function $\rho\left(t-t_{i}\right)$ was approximated by a Gaussian of width $\sigma=0.069$ ps. The index $i$ runs over all $N$ events. Finally, $t_{\mathrm{min}}$, the minimum proper lifetime allowed in the event selection was taken to be $0.5 \mathrm{ps}$. The direct $\mathrm{CP}$ violation term proportional to $\cos (\Delta \mathrm{mt})$ in $\mathrm{W}_{ \pm}(\mathrm{t})$ was neglected in the analyses to date, leaving the sinus of the $\mathrm{B}_{\mathrm{d}}$ mixing phase, $2 \beta$, the only free parameter in the fit.

ATLAS will achieve high sensitivity in this channel by dedicated triggers selecting $\mathrm{J} / \psi \rightarrow \mu^{+} \mu^{-}$and $\mathrm{J} / \psi \rightarrow \mathrm{e}^{+} \mathrm{e}^{-}$and by several tagging methods [11] using a muon or electron from the associated B-decay, or the charge of the jet that contains a signal B-decay. The expected event yields and the precisions obtained 
with different tagging methods are summarized in Table 1. Results are given for several trigger options. Maximal performance can be achieved combining the lower threshold di-muon trigger $\mu 6 \mu 3$ (the numbers correspond to the $p_{\mathrm{T}}$ cuts in $\mathrm{GeV}$ of the selected leptons) with the very low threshold trigger selecting $\mu 6$ at level-1 and a pair of electrons (e1e1) by a global track search at level-2. Combining all tags, a precision on $\sin (2 \beta)$ of 0.01 could be achieved after 3 years running at a luminosity of $10^{33} \mathrm{~cm}^{-2} \mathrm{~s}^{-1}$. The most conservative estimate was made using only the di-muon higher-threshold trigger $\mu 6 \mu 5$. The corresponding precision after 3 years and the same luminosity will be 0.016 . It was estimated that the corresponding systematic uncertainty would be 0.005 .

\section{Measurement of CP violation in $\mathrm{B}_{\mathrm{d}} \rightarrow \pi^{+} \pi^{-}$}

The decay channel $\mathrm{B}_{\mathrm{d}} \rightarrow \pi^{+} \pi^{-}$allows another angle of the unitarity triangle, $\alpha$, to be probed. Unfortunately, large penguin contributions suggested by theoretical calculations [12] and also indicated by the latest CLEO results [13], makes the interpretation of $\mathrm{B}_{\mathrm{d}} \rightarrow \pi^{+} \pi^{-}$measurements in terms of $\alpha$ difficult. In this channel the ATLAS analyses, $[14,15]$, were done in terms of the time-dependent CP asymmetry $\mathcal{A}_{\mathrm{CP}}$ :

$$
\mathcal{A}_{\mathrm{CP}} \equiv \frac{\Gamma(t)-\bar{\Gamma}(t)}{\Gamma(t)+\bar{\Gamma}(t)} \sim \mathcal{A}_{\mathrm{CP}}^{\mathrm{mix}} \sin (\Delta m t)+\mathcal{A}_{\mathrm{CP}}^{\mathrm{dir}} \cos (\Delta m t)
$$

separated into mixing-induced and direct $\mathrm{CP}$ violation parts, which are simply related to the weak phase of this decay. The CP asymmetry observables, $\mathcal{A}_{\mathrm{CP}}^{\text {mix }}$ and $\mathcal{A}_{\mathrm{CP}}^{\mathrm{dir}}$, are defined by the following equations:

$$
\mathcal{A}_{\mathrm{CP}}^{\mathrm{mix}}=2|P / T| \sin \delta \sin \alpha ; \mathcal{A}_{\mathrm{CP}}^{\mathrm{dir}}=-\sin (2 \alpha)-2|P / T| \cos \delta \cos (2 \alpha) \sin \alpha .
$$

These contain other unknown parameters: the ratio of penguin and tree amplitudes $|P / T|$ and a strong phase $\delta$. The low branching ratio and lack of sub-mass constraints make the reconstruction of $\mathrm{B}_{\mathrm{d}} \rightarrow \pi^{+} \pi^{-}$a demanding task. Additional problems are posed by isolating the signal from other two-body topologies, such as $\mathrm{B}_{\mathrm{d}} \rightarrow \mathrm{K}^{+} \pi^{-}, \mathrm{B}_{\mathrm{s}} \rightarrow \mathrm{K}^{+} \mathrm{K}^{-}, \mathrm{B}_{\mathrm{s}} \rightarrow \mathrm{K}^{-} \pi^{+}, \Lambda_{\mathrm{b}}^{0} \rightarrow p \pi^{-}$and $\Lambda_{\mathrm{b}}^{0} \rightarrow p K^{-}$ decays. ATLAS will use a maximum likelihood fit to extract CP asymmetry parameters for $\mathrm{B}_{\mathrm{d}} \rightarrow \pi^{+} \pi^{-}$defined in equation (4) simultaneously with asymmetry parameters of other two-body classes.

Fig.4a shows the sensitivity to $\alpha$ as a function of $2 \alpha$ for $\delta=10^{\circ},|P / T|=0.4$ and three uncertainties values for $|P / T|, 0 \%, 30 \%$ and $100 \%$. The precision on $\alpha$ varies strongly with the parameters. For $\alpha$ around $90^{\circ}$ and with a theoretical uncertainty in $|P / T|$ of $30 \%$ ATLAS should be able to measure $\alpha$ with an error of $16^{\circ}$. Summarising the total LHC capacity of LHCb, CMS and ATLAS after one year (Fig.4b) it appears that for values of $\alpha$ around $90^{\circ}$ the sensitivity to $\alpha$ is already limited after one year if the theoretical uncertainty on $|P / T|$ is not better than $30 \%$. 
(a)

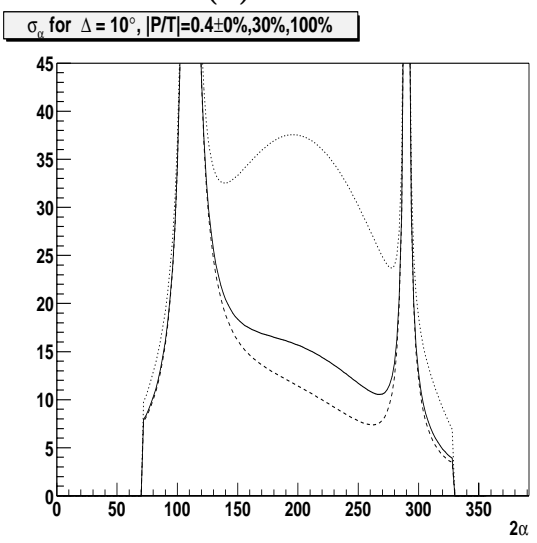

(b)

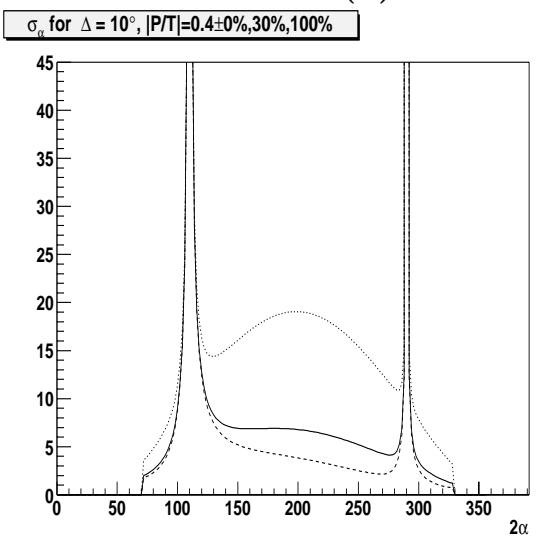

Fig. 4. (a) ATLAS precision for measuring $\alpha$ from $\mathrm{B}_{\mathrm{d}} \rightarrow \pi^{+} \pi^{-}$as a function of $2 \alpha$ after three years of $10^{33} \mathrm{~cm}^{-2} \mathrm{~s}^{-1}$ data-taking. The strong phase $\delta$ was fixed at $10^{\circ}$. The three lines correspond to $|P / T|=0.2 \pm 30 \%$ ( full line), $|P / T|=0.2 \pm 0 \%$ (dashed line), $|P / T|=0.2 \pm 100 \%$ (dotted line). (b) Analogous results for the complete LHC potential after 1 year of running, including LCHb, ATLAS and CMS in a simultaneous fit.

\section{Physics of $\mathrm{B}_{\mathrm{s}}^{0}$ mesons}

\subsection{Oscillation measurements}

The $\mathrm{B}_{\mathrm{s}}^{0}$ and $\overline{\mathrm{B}}_{\mathrm{s}}^{0}$ mesons mix due to weak interactions. The $\mathrm{B}_{\mathrm{s}}^{0}-\overline{\mathrm{B}}_{\mathrm{s}}^{0}$ system is characterized by two physics eigenstates with different masses and decay rates. Both the mass difference $\Delta \mathrm{M}_{\mathrm{s}}$ and the rate difference $\Delta \Gamma_{\mathrm{s}}$ can be described in the Standard Model (SM) by second order weak processes with $\Delta B=2$. Their experimental determination will be a valuable input for flavour dynamics both in SM and for its possible extensions.

It has been proven that both variables are accessible by measurement in ATLAS. The mass difference $\Delta \mathrm{M}_{\mathrm{s}}$ will be measured by $\mathrm{B}_{\mathrm{s}}^{0}$ decays to flavour specific states $\mathrm{B}_{\mathrm{s}}^{0} \rightarrow \mathrm{D}_{\mathrm{s}} \pi, \mathrm{B}_{\mathrm{s}}^{0} \rightarrow \mathrm{D}_{\mathrm{s}} \mathrm{a}_{1} . \Delta \Gamma_{\mathrm{s}}$ can be precisely measured in $\mathrm{B}_{\mathrm{s}}^{0} \rightarrow$ $\mathrm{J} / \psi \phi$.

\section{2 $\Delta \mathrm{M}_{\mathrm{s}}$ measurement with $\mathrm{B}_{\mathrm{s}}^{0} \rightarrow \mathrm{D}_{\mathrm{s}} \pi, \mathrm{B}_{\mathrm{s}}^{0} \rightarrow \mathrm{D}_{\mathrm{s}} \mathrm{a}_{1}$.}

In $\mathrm{B}_{\mathrm{s}}^{0} \rightarrow \mathrm{D}_{\mathrm{s}} \pi, \mathrm{B}_{\mathrm{s}}^{0} \rightarrow \mathrm{D}_{\mathrm{s}} \mathrm{a}_{1}$, the probability $p_{-}$that an initially (time $\mathrm{t}=0$ ) pure $\mathrm{B}_{\mathrm{s}}^{0}$ will be observed as a $\overline{\mathrm{B}}_{\mathrm{s}}^{0}$ and the probability $p_{+}$that it will remain a $\mathrm{B}_{\mathrm{s}}^{0}$ are described in terms of $\Gamma, \Delta \Gamma_{\mathrm{s}}$ and $\Delta \mathrm{M}_{\mathrm{s}}$ by following formulae:

$$
p_{\mp}(t)=e^{-\Gamma t}\left(\cosh \frac{\Delta \Gamma_{\mathrm{s}}}{2} t \mp \cos \Delta \mathrm{M}_{\mathrm{s}} t\right) \frac{\Gamma^{2}-\Delta \Gamma_{\mathrm{s}}{ }^{2}}{2 \Gamma}
$$


The parameter $\Delta \mathrm{M}_{\mathrm{S}}$ then can be derived from the ratio:

$$
r(t)=\frac{p_{+}(t)-p_{-}(t)}{p_{+}(t)+p_{-}(t)}=\frac{\cos \Delta \mathrm{M}_{\mathrm{s}} t}{\cosh \frac{\Delta \Gamma_{\mathrm{s}}}{2} t}
$$

which is diluted in the case of $\Delta \Gamma_{\mathrm{s}} \neq 0$ by the time dependent hyperbolic function. It was shown however that for $\Delta \Gamma_{\mathrm{s}} / \Gamma \leq 0.2$ no significant change in the sensitivity range of $\Delta \mathrm{M}_{\mathrm{S}}$ is expected. The ATLAS performance parameters for the $\mathrm{B}_{\mathrm{s}}^{0} \rightarrow \mathrm{D}_{\mathrm{s}} \pi, \mathrm{B}_{\mathrm{s}}^{0} \rightarrow \mathrm{D}_{\mathrm{s}} \mathrm{a}_{1}$ processes and the background were determined by detector simulations and corresponding parameters were used as input to a fit in repeated Monte Carlo experiments. A summary of the results is given in Table 2, extracted from [11]. It is clear that already after 1 year at a luminosity of $10^{33} \mathrm{~cm}^{-2} \mathrm{~s}^{-1}$ ATLAS can fully explore the SM allowed range of $\Delta M_{s}$.

\begin{tabular}{|c|c|}
\hline$B_{s}^{0}$ channels & $D_{s}^{-} \pi^{+}, D_{s}^{-} a_{1}^{+}$ \\
$D_{s}^{-}$channel & $\phi\left(K^{+} K^{-}\right) \pi^{-}$ \\
$a_{1}^{+}$channel & $\rho^{0} \pi^{+}$ \\
\hline signal events in 1 year & 3457 \\
proper time resolution & $50 \mathrm{fs}(60.5 \%)$ \\
(Gaussian functions) & $93 \mathrm{fs}(39.5 \%)$ \\
\hline$\Delta M_{s}$ 95\% CL 1 year & $30 \mathrm{ps}^{-1}$ \\
$x_{s}$ reach 95\% CL 1 year & 46 \\
\hline
\end{tabular}

Table 2. Summary of $\mathrm{B}_{\mathrm{s}}^{0}$ oscillation frequency measurement in the channels $\mathrm{B}_{\mathrm{s}}^{0} \rightarrow \mathrm{D}_{\mathrm{s}} \pi, \mathrm{B}_{\mathrm{s}}^{0} \rightarrow \mathrm{D}_{\mathrm{s}} \mathrm{a}_{1}$

The precision of $\Delta \Gamma_{\mathrm{s}}$ measurement from $\mathrm{B}_{\mathrm{s}}^{0} \rightarrow \mathrm{D}_{\mathrm{s}} \pi$ and $\mathrm{B}_{\mathrm{s}}^{0} \rightarrow \mathrm{D}_{\mathrm{s}} \mathrm{a}_{1}$ using the method suggested by Dunietz [16] was estimated. It was shown [17] that these processes do not provide a useful $\Delta \Gamma_{\mathrm{s}}$ measurement in ATLAS.

\section{3 $\Delta \Gamma_{\mathrm{s}}$ and $\mathrm{B}_{\mathrm{s}}^{0}$ mixing phase $\phi_{s}$ from $\mathrm{B}_{\mathrm{s}}^{0} \rightarrow \mathrm{J} / \psi \phi$}

The $\mathrm{B}_{\mathrm{s}}^{0} \rightarrow \mathrm{J} / \psi \phi$ decay leads to three final state helicity configurations and their linear combinations are CP eigenstates with different CP parities [16]. This means that it is not possible to extract a CP-violating weak mixing phase $\phi_{s}=\arg \left(V_{c s}^{*} V_{c b} / V_{c s} V_{c b}^{*}\right)$ if the helicity amplitudes are not separated. The experimental observables are three independent angles and the $\mathrm{B}_{\mathrm{s}}^{0}$ proper time of the decay $\mathrm{B}_{\mathrm{s}}^{0} \rightarrow \mathrm{J} / \psi \phi \rightarrow \mu^{+} \mu^{-} \mathrm{K}^{+} \mathrm{K}^{-}$. In some cases the initial $\mathrm{B}_{\mathrm{s}}^{0}$ flavour can also be tagged. A determination of the background contribution and its physics characteristics brings other important information. The ATLAS precision for these measurements was determined by detector response simulations and was used as the input to angular analyses based on a maximum likelihood fit in repeated Monte Carlo experiments [18]. The difference of the mass eigenstate decay rates, $\Delta \Gamma_{\mathrm{s}}$, their average value $\Gamma_{s}$ and the weak phase $\phi_{s}$ were simultaneously determined along with the two helicity amplitude values and their strong phases. The mixing parameter $x_{s} \equiv \Delta M_{s} / \Gamma_{s}$ was assumed to be measured in $\mathrm{B}_{\mathrm{s}}^{0} \rightarrow \mathrm{D}_{\mathrm{s}} \pi$ and $\mathrm{B}_{\mathrm{s}}^{0} \rightarrow \mathrm{D}_{\mathrm{s}} \mathrm{a}_{1}$ events and was fixed. While all eight parameters 


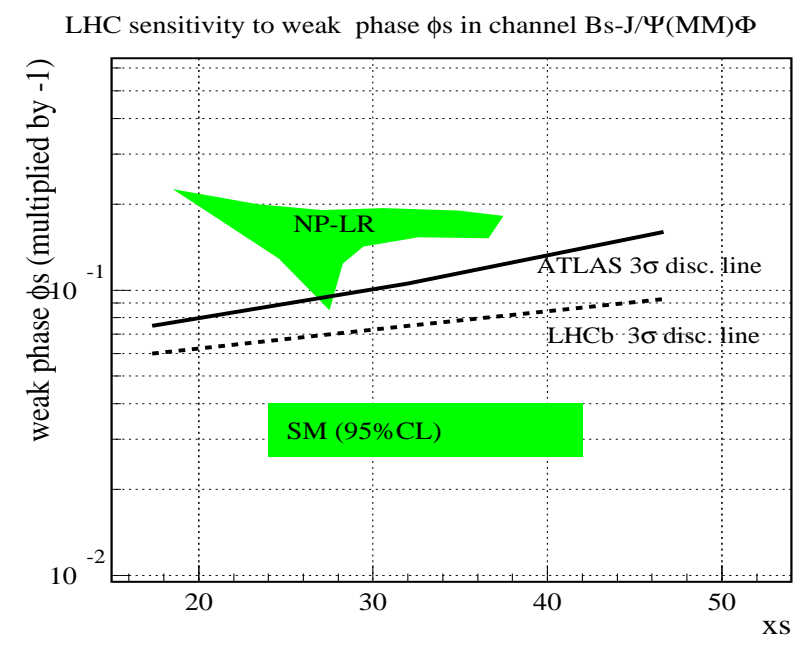

Fig. 5. The $x_{s}-\phi_{s}$ region allowed by the SM, Left-right symmetric models (NPLR), the region of experimental sensitivity of ATLAS (full line) and of LHCb in the same process (hashed line).

are independent in the theoretical models, the experimental resolution causes some to become correlated. The highest correlation appeared between the two strong phases and prevented their simultaneous determination. Thus, in the final analysis the two parameters were fixed.

The analyses of this channel were updated recently [19] for the initial inner detector layout and thus the results on precision of parameters are given within the new scope. The di-muon trigger was assumed to select the events with $p_{\mathrm{T}}$ larger than $6 \mathrm{GeV}$ for the first muon and $3 \mathrm{GeV}$ for the second muon. After 3 years at luminosity $10^{33} \mathrm{~cm}^{-2} \mathrm{~s}^{-1}$ the value of $\Delta \Gamma_{\mathrm{s}}$ can be determined with a relative error of $12 \%$. The precision of the $\phi_{s}$ determination depends on the value of $x_{s}$ and on the proper-time resolution. The ATLAS discovery line in the $\left(x_{s}-\phi_{s}\right)$ plane is displayed in Fig.6.3, together with regions allowed by the SM and by an example of new physics model [20]. The ATLAS precision is high enough to be sensitive to new physics.

\section{$7 \quad$ Rare decays prospects}

Flavour changing neutral current decays $b \rightarrow s, b \rightarrow d$ which occur only at loop level in the SM have small exclusive branching ratios $B r<\mathcal{O}\left(10^{-5}\right)$. They are sensitive to new physics. Within the SM, these decays are sensitive to the CKM matrix elements $\left|V_{t d}\right|,\left|V_{t s}\right|$.

In the era before LHC, some rare decays are accessible at $\mathrm{e}^{+} \mathrm{e}^{-}$factories and the Tevatron. In particular for $\mathrm{B} \rightarrow \mathrm{K}^{*} \gamma$ at time of LHC quite accurate 
measurements should be available. The process $\mathrm{B} \rightarrow \mathrm{K}^{*} \mu \mu$ can be seen, however the mass and angular distributions can only be studied at LHC. Purely muonic rare decays can be observed before LHC only if they are drastically enhanced comparing to the $\mathrm{SM}$ predictions $\operatorname{Br}\left(\mathrm{B}_{\mathrm{s}}^{0} \rightarrow \mu^{+} \mu^{-}\right)=(3.5 \pm 1.0) \cdot 10^{-9}$ and $\operatorname{Br}\left(\mathrm{B} \rightarrow \mu^{+} \mu^{-}\right)=(1.5 \pm 1.0) \cdot 10^{-10}[21]$.

\subsection{Purely muonic decays}

Using the simulation of the detector response, ATLAS has demonstrated that purely muonic decays can be selected by the trigger and reconstructed in the offline analyses at both low and high luminosities expected to be $10^{33} \mathrm{~cm}^{-2} \mathrm{~s}^{-1}$ and $10^{34} \mathrm{~cm}^{-2} \mathrm{~s}^{-1}$ respectively. It was assumed that the performance of the inner detector will not be degraded for the higher luminosity case. Expected signal and background statistics for $\mathrm{B}_{\mathrm{s}}^{0} \rightarrow \mu^{+} \mu^{-}$and $\mathrm{B}_{\mathrm{d}}^{0} \rightarrow \mu^{+} \mu^{-}$are summarized in Table 3, extracted from [11] and [22].

\begin{tabular}{|c|c|c|c|}
\hline & $\begin{array}{c}\text { Signal } \\
\mathrm{B}_{\mathrm{s}}^{0} \rightarrow \mu^{+} \mu^{-}\end{array}$ & $\begin{array}{c}\text { Signal } \\
\mathrm{B}_{\mathrm{d}}^{0} \rightarrow \mu^{+} \mu^{-}\end{array}$ & BG \\
\hline 1 year $10^{34} \mathrm{~cm}^{-2} \mathrm{~s}^{-1}$ & 92 & 14 & 660 \\
\hline 3 years at $10^{33} \mathrm{~cm}^{-2} \mathrm{~s}^{-1}$ & 27 & 4 & 93 \\
\hline
\end{tabular}

Table 3. Expected signal and background numbers of reconstructed $\mathrm{B}_{\mathrm{s}}^{0} \rightarrow \mu^{+} \mu^{-}$ and $\mathrm{B}_{\mathrm{d}}^{0} \rightarrow \mu^{+} \mu^{-}$events.

Already after one year of high luminosity running ATLAS will be able to observe $\mathrm{B}_{\mathrm{s}}^{0} \rightarrow \mu^{+} \mu^{-}$and measure its branching ratio and perform a high sensitivity search for $\mathrm{B}_{\mathrm{d}}^{0} \rightarrow \mu^{+} \mu^{-}$.

\subsection{Semi-muonic decays}

Assuming the SM to be valid, the measurement of the branching fractions of the decays $\mathrm{B}_{\mathrm{d}}^{0} \rightarrow \rho^{0} \mu^{+} \mu^{-}$and $\mathrm{B}_{\mathrm{d}}^{0} \rightarrow \mathrm{K}^{* 0} \mu^{+} \mu^{-}$gives, in principle, the possibility to extract the ratio of CKM elements $\left|V_{t d}\right|,\left|V_{t s}\right|$. Using detector simulations ATLAS has estimated that the branching fraction ratio can be measured with a statistical precision of $14 \%$; however as pointed in [14] the extraction of $\left|V_{t d}\right|$ $/\left|V_{t s}\right|$ is limited by theoretical uncertainty in the ratio of the form factors describing $\mathrm{B}_{\mathrm{d}}^{0} \rightarrow \mathrm{K}^{* 0} \mu^{+} \mu^{-}$and $\mathrm{B}_{\mathrm{d}}^{0} \rightarrow \rho^{0} \mu^{+} \mu^{-}$decays.

ATLAS studied the prospects of measurements of the forward-backward asymmetry in $\mathrm{B}_{\mathrm{d}}^{0} \rightarrow \mathrm{K}^{* 0} \mu^{+} \mu^{-}$:

$$
A_{\mathrm{FB}}(\hat{s})=\frac{1}{d \Gamma / d \hat{s}}\left(\int_{0}^{1} \frac{d^{2} \Gamma}{d \hat{s} d \cos (\theta)} d \cos (\theta)-\int_{-1}^{0} \frac{d^{2} \Gamma}{d \hat{s} d \cos (\theta)} d \cos (\theta)\right)
$$

where $\theta$ is the angle between the $\mu^{+}$and the $B$ meson direction in the rest frame of the muon pair; $\hat{s}=s / m_{\mathrm{B}}^{2}, s=q^{2}, q=p^{+}+p^{-}$where $p^{ \pm}$are four-momenta 


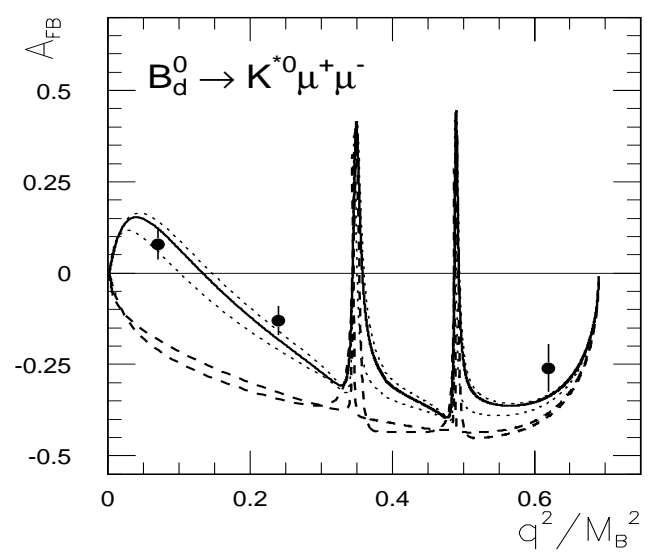

Fig. 6. Sensitivity of asymmetry measurement in $B_{d}^{0} \rightarrow K^{* 0} \mu^{+} \mu^{-}$. Points are the simulation results in three di-muon invariant mass regions. The solid line is SM and dotted and dashed lines are MSSM predictions with different Wilson coefficients $C_{7 \gamma}$ (for details see [11]).

of the two muons. The precision of $A_{F B}$ was estimated in three regions of dimuon invariant mass. The data are presented in Fig. 6, taken from [11], together with asymmetry values in the SM and an example of a super-symmetric model. The precision of ATLAS will be sufficient to distinguish between the SM and its extension using the measurements in the first region.

\section{Beauty production and QCD tests}

The LHC will probe kinematic regions of strong interactions that have not yet been explored. Compared with previous hadron experiments this will be a higher collision energy, $14 \mathrm{TeV}$, and over a wider Bjorken $x$ region (ATLAS will be sensitive down to $x \sim 10^{-4}[23]$ ). The high beauty cross section at LHC will allow b-production measurement to be extended up to transverse momenta of several hundred $\mathrm{GeV}$ and correlations between $\mathrm{b}$ and $\overline{\mathrm{b}}$ quarks to be investigated. It will be also possible to study multiple heavy flavour production, which was difficult to access in previous experiments due to limited statistics. QCD is nowadays recognized as a well proven theory and new data are expected to determine the boundaries within which the perturbation theory provides an adequate description.

Numerous CDF and D0 measurements [24] yielded a cross section for single-b quark production approximately 2 times higher then calculated in NLO QCD. The RUN-I statistics were not sufficient to fully explore $b \bar{b}$ correlations. Using semi-inclusive B-decay modes, [25, 26], required an application of isolation cuts leading finally to an information loss in the configurations where the $\mathrm{b}$ and $\overline{\mathrm{b}}$ quarks were produced close to each other. This region is sensitive to higher 

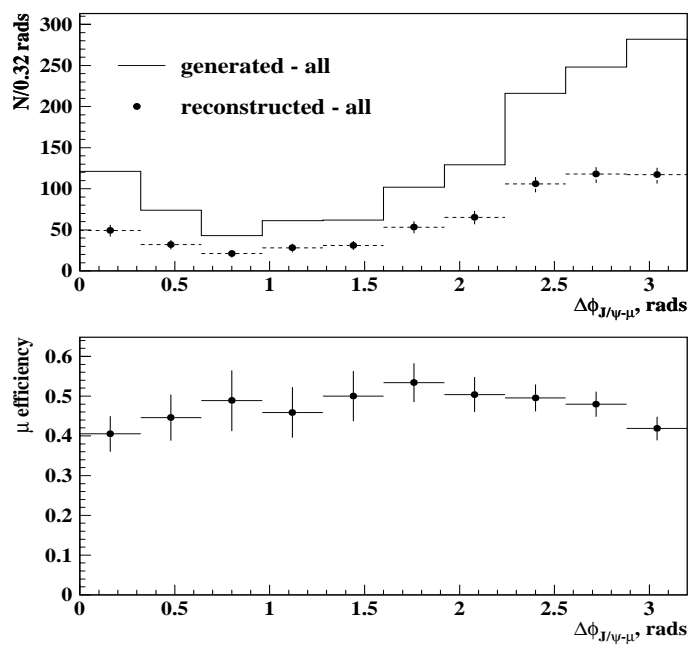

Fig. 7. ATLAS simulation of $\mathrm{b} \overline{\mathrm{b}}$ correlation measurements using $\mathrm{B}_{\mathrm{d}}\left(\mathrm{J} / \psi\left(\mu^{+} \mu^{-}\right) \mathrm{K}^{0}\right)+\mu \mathrm{X}$ events. The azimuthal angle difference $\Delta \phi(\mathrm{J} / \psi-\mu)$ for generated (full line) and reconstructed (points) events (upper figure), and the corresponding reconstruction efficiency (lower figure).

order QCD contributions [27].

The ATLAS performance studies were done for two channels selected to measure the azimuthal angle difference $\Delta \phi(\mathrm{b} \overline{\mathrm{b}})$ between $\mathrm{b}$ and $\overline{\mathrm{b}}$ quarks:

$$
\begin{gathered}
\overline{\mathrm{b}} \rightarrow \mathrm{B}_{\mathrm{d}} \rightarrow \mathrm{J} / \psi(\mu \mu) \mathrm{K}^{0}, \mathrm{~b} \rightarrow \mu \mathrm{X} \\
\overline{\mathrm{b}} \rightarrow \mathrm{B}_{\mathrm{s}} \rightarrow \mathrm{J} / \psi(\mu \mu) \phi, \mathrm{b} \rightarrow \mu \mathrm{X} .
\end{gathered}
$$

The numbers of events expected after 3 years at $10^{33} \mathrm{~cm}^{-2} \mathrm{~s}^{-1}$ are $4.8 \cdot 10^{4}$ and $3.2 \cdot 10^{4}$ respectively. No isolation cuts were needed to separate the exclusively reconstructed B decay from the semi-leptonic muon, and the reconstruction efficiency remained high in topologies where the azimuthal angle difference $\Delta \phi(\mathrm{J} / \psi-\mu)$ between the $\mathrm{J} / \psi$ and the $\mu$ was small, see Fig.7, [10].

Special attention was devoted to background events in which the muon is produced from the decays $\mathrm{K}^{ \pm}, \pi^{ \pm} \rightarrow \mu^{ \pm} \mathrm{X}$ instead of $\mathrm{b} \rightarrow \mu \mathrm{X}$. The study showed that this background is not problematic in $\mathrm{B}_{\mathrm{d}}$ decays, however it is important in the case of the $\mathrm{B}_{\mathrm{s}}^{0}$ meson. This particle is composed of $\mathrm{b}$ and $\mathrm{s}$ quarks and so is always accompanied by the associated strange quark, which mostly hadronizes to a $\mathrm{K}$ meson. The characteristic feature of this background is that the muon from the $\mathrm{K}$ decay is correlated with the $\mathrm{B}_{\mathrm{s}}^{0}$ meson. After applying cuts to reject $\mathrm{K}^{ \pm}, \pi^{ \pm} \rightarrow \mu^{ \pm} \mathrm{X}$ the contribution of this background is still important especially for high $p_{\mathrm{T}}$ events, $p_{\mathrm{T}}\left(\mathrm{B}_{\mathrm{s}}^{0}\right)>50 \mathrm{GeV}$, see Fig.8, taken from [23]. The results of this study show the importance of simultaneous measurements of b $\bar{b}$ correlations and of the production of $\mathrm{b} \overline{\mathrm{b}} \overline{\mathrm{s}}$ combinations.

The beauty production studies will be extended to semi-inclusive events containing $\mathrm{b} \overline{\mathrm{b}} \rightarrow \mathrm{J} / \psi\left(\mu^{+} \mu^{-}\right) \mathrm{X}$ and to b-jets to access the high- $p_{\mathrm{T}}$ region. 

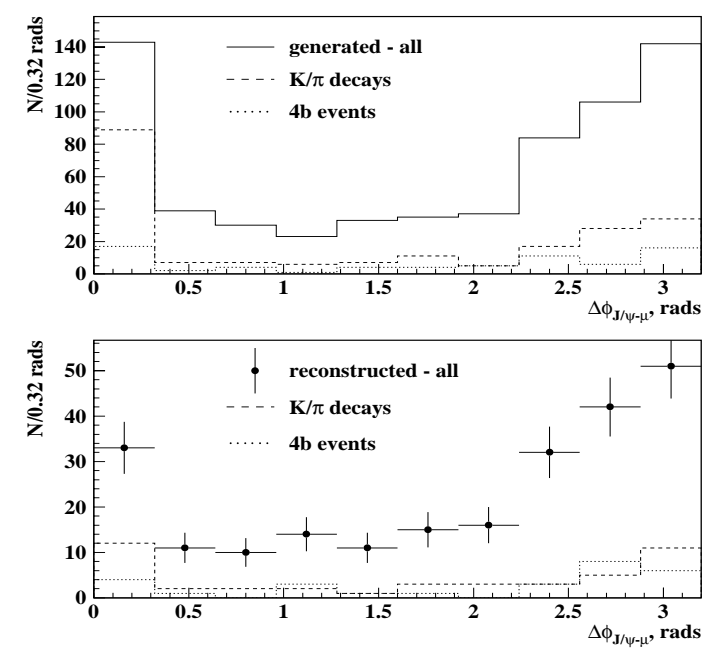

Fig. 8. ATLAS simulation of $\mathrm{b} \overline{\mathrm{b}}$ correlation measurements using $\mathrm{B}_{\mathrm{s}}\left(\mathrm{J} / \psi\left(\mu^{+} \mu^{-}\right) \phi\right)+\mu \mathrm{X}$ events. The distributions of azimuthal angle difference $\Delta \phi(\mathrm{J} / \psi-\mu)$ for generated (upper figure) and reconstructed (lower figure) events.

\section{Conclusions}

Various ATLAS studies have clearly shown that the detector is well equipped for a multi-thematic B-physics program. The ATLAS B-physics trigger strategy has been revised to maximize the physics potential within tight funding constraints. It will rely on a di-muon trigger for the initial luminosity of $2 \cdot 10^{33} \mathrm{~cm}^{-2} \mathrm{~s}^{-1}$ and will extend selections at lower luminosities when there is spare processing capacity.

In the physics of $\mathrm{CP}$ violation the main emphasis is on underlying mechanisms and the evidence for new physics. ATLAS can make a particularly precise measurement of the unitarity triangle angle $\beta$. Apart from the B-factories 'benchmark' $\mathrm{B}_{\mathrm{d}}$ decay modes, the LHC 'gold-plated' mode $\mathrm{B}_{\mathrm{s}}^{0} \rightarrow \mathrm{J} / \psi \phi$ was studied. $\mathrm{B}_{\mathrm{s}}^{0}$ physics studies made it clear that there is a sensitivity to a mass difference $\Delta \mathrm{M}_{\mathrm{S}}$ far beyond $\mathrm{SM}$ expectations. The rate difference $\Delta \Gamma_{\mathrm{s}}$ can be best measured in ATLAS from $\mathrm{B}_{\mathrm{s}}^{0} \rightarrow \mathrm{J} / \psi \phi$. Important for rare decays is the possibility of continuing the measurements at full LHC luminosity, when $\mathrm{B}_{\mathrm{s}}^{0} \rightarrow \mu^{+} \mu^{-}$with a SM ratio $10^{-9}$ can already be observed after one year. Precision measurements can be made for $\mathrm{B}_{\mathrm{d}}^{0} \rightarrow \mathrm{K}^{* 0} \mu^{+} \mu^{-}$. Large statistics of exclusive or semi-inclusive B-decay channels, especially those with $\mathrm{J} / \psi \rightarrow \mu^{+} \mu^{-}$, allow QCD tests of central b-production at LHC, in particular the correlations between $b$ and $\bar{b}$. The ATLAS potential for B-physics has not been fully explored yet and other studies are ongoing. 


\section{References}

1. G.H. De Monchenault (for the Babar collaboration), CP violation: Recent results from Babar, SLAC-PUB-9816, DAPNIA-03-111, May 2003. 8pp.

2. M. Yamauchi (for the Belle collaboration), Nucl.Phys.Proc.Suppl.117:83-97,2003

3. M. Bishai (for the CDF collaboration), Beauty results at CDF RUN II, IV International Symposium on LHC Physics and detectors, Fermilab, May 1-3 2003.

4. G. Borissov (for the D0 collaboration), B-physics at D0, IV International Symposium on LHC Physics and detectors, Fermilab, May 1-3 2003.

5. J. Baines (for the ATLAS Collaboration) Nuclear Physics B 120 (2003) 139-144.

6. ATLAS High-Level Triggers, DAQ and DCS Technical Proposal, CERN LHCC 2000-17.

7. ATLAS Inner Detector Technical Design Report, CERN, LHCC 97-16.

8. ATLAS Detector Performance Technical Design Report, Vol.1, CERN LHCC 99-14.

9. ATLAS First-level Trigger Technical Design Report, CERN LHCC 98-14.

10. S. Robins, Measuring b-b correlations in ATLAS, ATL-PHYS-2000-026.

11. ATLAS Detector Performance Technical Design Report, Vol.2, CERN LHCC 9915.

12. M.Gronau, Phys.Lett.B300, 163(1993),

A. Ali, G.Kramer, C.D.Lu, Phys.Rev.D59,014005(1999).

13. D.E.Jaffe ( CLEO coll.), hep-ex/9910055.

14. 1999 CERN Workshop on Standard Model Physics (and more) at the LHC, CERN 2000-004.

15. D.Rousseau, private communication.

16. I. Dunietz, Phys. Rev. D52(1995) 3048.

17. M. Smizanska, Determination of $\Delta \Gamma_{\mathrm{s}}$ by ATLAS and the impact of non-zero $\Delta \Gamma_{\mathrm{s}}$ on other Bs studies, ATL-PHYS-99-003.

18. M. Smizanska, Nucl.Instrum.Meth.A446:138-142,2000.

19. E. Bouhova, private communication.

20. P. Ball, R. Fleischer, Phys.Lett. B475 (2000) 111-119; D. Silverman, Phys. Rev. D58(1998) 095006.

21. A. Ali, J.Phys.G18:1605-1626,1992.

22. D. Melikov, F. Rizatdinova, S.Sivoklokov, L. Smirnova, Studying the physics beyond the Standard Model in rare semileptonic $\mathrm{B}_{\mathrm{d}}^{0} \rightarrow \mathrm{K}^{* 0} \mu^{+} \mu^{-}$with ATLAS detector, ATL-PHYS-94-045.

23. S. Frixione et al., J.Phys.G27:1111-1157,2001.

24. D0 Collab., S. Abachi et al., CDF Collab., F. Abe et al.,1996, Phys. Lett. 370 B 2391995 Phys. Rev. Lett. 75 1451, 1996 Phys. Rev. D 531051.

25. D0 Collab., S. Abachi et al., 1996. Phys. Lett. 370 B 239

26. CDF Collab., F. Abe et al., 1996. Phys. Rev. D 531051

27. S.P. Baranov, M. Smizanska, Phys.Rev.D62 (2000) 014012. 